\title{
Over 130 lm/W All-Phosphorescent White OLEDs for Next-generation Lighting
}

\author{
Kazuki Kato, Toshihiko Iwasaki, and Takatoshi Tsujimura \\ OLED Unit, Advanced Layers Business Unit, \\ KONICA MINOLTA, INC. \\ 2970 Ishikawa-machi, Hachioji-shi, Tokyo 192-8505, Japan \\ kazuki.kato@konicaminolta.com
}

\begin{abstract}
OLED lighting is the promising technology that makes the future lighting more valuable one. For OLED lighting to penetrate into market, considerable challenges remain. From ecology perspective, it is critical to improve the luminous efficacy. White OLED device with the world highest record of $1391 \mathrm{~m} / \mathrm{W}(\mathrm{CCT}=2857 \mathrm{~K})$ at 1,000 $\mathrm{cd} / \mathrm{m}^{2}$ was successfully achieved by introducing proprietary blue phosphorescent emitter to raise the internal quantum efficiency and improved internal extraction structure to enhance the light out-coupling efficiency.
\end{abstract}

Keywords: high luminous efficacy, blue phosphorescent emitter, internal extraction structure, white OLEDs

\section{Introduction}

Since so called " 1 st OLED device" was reported by Ching Tang in 1987 [1], performance of OLED device has made significant improvement in this quarter century. Though, before Tang's paper, the OLED used to require very high voltage for emission like more than $100 \mathrm{~V}$, the state-of-the-art OLED device has very high luminous efficacy enough to be used for various displays including television [2], and even for cellular phone displays, for which very low power consumption is required. The luminous efficacy has been improved enough for OLED displays to compete with other technologies. However, for lighting applications, the luminous efficacy requirement is more stringent, especially when energy saving is stressed these days.

The luminous efficacy of the OLED device is proportionate to the external quantum efficiency (EQE), which can be split into the internal quantum efficiency (IQE) and the out-coupling efficiency (OCE). Therefore, for the efficient improvement, it is important to increase both the IQE by a material or layer stack design technology and the OCE by optical design and out-coupling technologies.

Firstly, optimization of career balance with adjusting energy level and mobility of stacked layers such as emitting, transportation, injection, or blocking layer is required to maximize the IQE, especially for an effective hole-electron recombination.

According to spin statics, the recombination yields singlet and triplet excitons by a ratio of $1: 3$ respectively. It is well known that phosphorescent OLED enables radiation from the triplet exciton and its IQE has already reached nearly $100 \%$ [3]. Although an all-phosphorescent OLED device provides very high IQE, a blue phosphorescent system tends to show a trade-off between color coordinate and lifetime.

Secondly, the OCE is only about $42 \%$ and overall panel efficiency is only $23 \%$ [4], because of mismatches of refractive indices among OLED 
materials, anode materials and substrate.

Many approaches have been proposed to improve the OCE using technologies such as scattering layers [5], corrugated substrates [6], out-coupling film [7], high refractive index substrates [8], and photonic crystals [9].

After the release of the world's first all-phosphorescent OLED panel, "symfos OLED-010K" in 2011 [10] and the presentation of white OLEDs of $81 \mathrm{~lm} / \mathrm{W}$ in 2013 [11], we have put effort into the development of high efficiency white OLEDs from various technological standpoints.

In this paper, key technologies including all-phosphorescent white OLEDs technology and light out-coupling technology to achieve higher efficacy is discussed.

\section{Key technologies for higher efficacy}

\subsection{Phosphorescent blue emitter}

From the view point of OLED materials for improvement of IQE, phosphorescent OLED technologies with intrinsically high quantum yields are expected. However, there is a limitation of emission color and lifetime of blue phosphorescent emitters so far. Generally, organic molecules secure molecular stability by extending their conjugations.

However, since the band gap of a phosphorescent blue emitter must be wide, the molecular structure should be composed of a relatively short conjugated system. Therefore, it is difficult to achieve a robust blue phosphorescent molecule in the OLED device. In addition, since it has a higher triplet energy and a longer excited state, it can be quenched easily even by a very small quantity of quencher existing in the system. For the above reasons, improving the stability of phosphorescent blue materials is essential to extend the OLED lifetime.

To overcome these issues, proprietary blue emitter system was studied through the original method in molecular design and the simulation of electronic state of molecules.

In tests before and after driving a device using a light-blue phosphorescent material, we found that degradation of material itself did not necessarily cause the device to degrade. We also found that the lifetime highly depends on a combination of emitter and host material chosen (Fig. 1). With a proper combination of emitter and host material, high efficiency and long lifetime

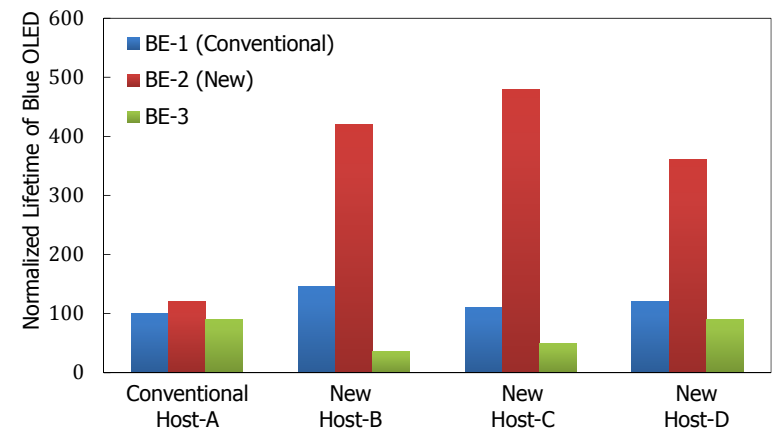

Fig. 1. Relative lifetime of various combinations of hosts (A, B, C \& D) and blue emitters (BE-1,-2 \& -3$)$

were achieved simultaneously.

As the result shown in Fig. 2, we achieved a highly efficient, long lived light-blue phosphorescent OLED (EQE 23\%, LT50= 100,000hours@300 cd/m²) [12].

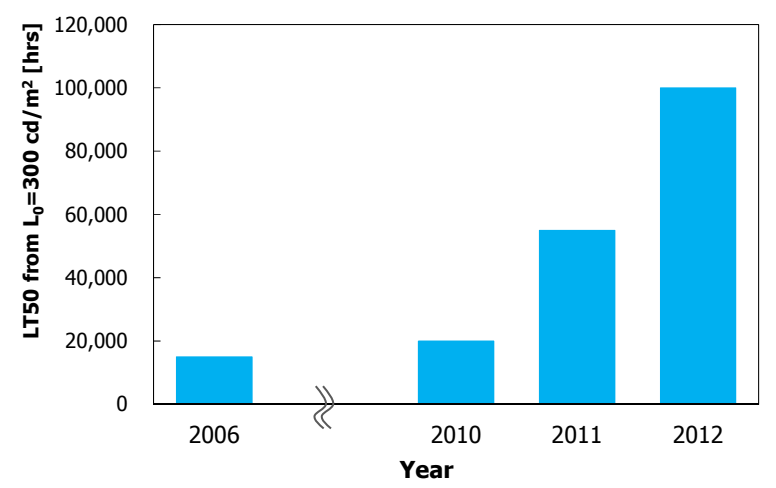

Fig. 2. Progress of KONICA MINOLTA light-blue phosphorescent OLED lifetime

This combination technology of host dopant, which technology was found in the light-blue materials, was applied to a deep-blue material development (Fig. 3).

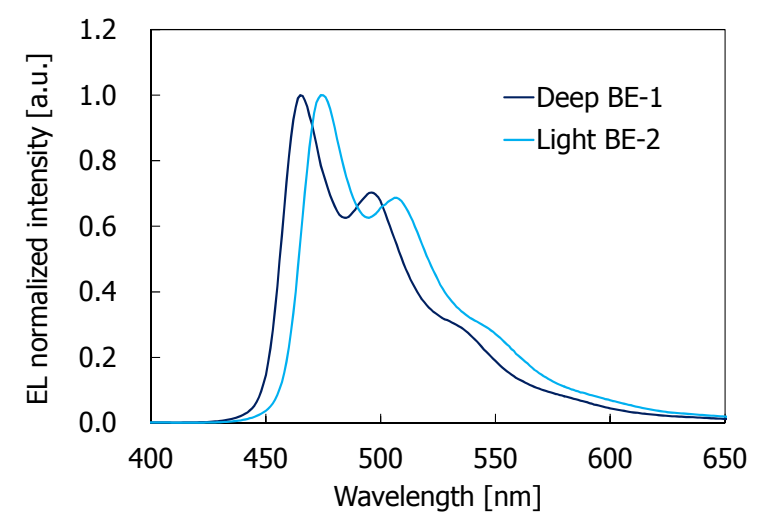

Fig. 3. Emission spectra of current light-blue and improved deep-blue phosphorescent emitters 


\subsection{Light extraction technology}

The root cause of the light loss is mismatches of refractive indices among OLED materials, an anode material and a substrate (Fig. 4). Part of the emission energy occurred in emission layer in OLED is lost by a coupling to the surface plasmon-polariton mode on the surface of a cathode metal near the emission state. This is called as the plasmon loss mode. Among the rest of the lost energy, a light element lower than the critical angle of the total reflection cannot escape from the organic stack and transparent electrode because of the large difference of the refractive indices between stacks and substrate (a waveguide mode). Also, part of the escaped energy from the stacks and transparent electrode, the lower angle element, cannot escape from the substrate because of the large difference of the refractive indices between substrate and air (a substrate mode). Finally, the rest of the energy is extracted as the emitted light (an air mode). Without any out-coupling technology, approximately only $20 \%$ of emission energy occurred in the emission layer can be extracted as the air mode.

To enhance the OCE, the majority of approach falls into three categories:

(1) External light extraction: surface modification at the substrate/air interface including substrate shaping, scattering layers and micro-lens patterns,

(2) Internal light extraction: refractive index matching layer, photonic crystal, and buckling structure, and

(3) Cathode refinement: patterning, topography and microstructures to inhibit plasmonic losses at the metal cathode surface.

The common theme of all these approaches is to avoid smooth interface to suppress total internal reflection and achieve better OCE.

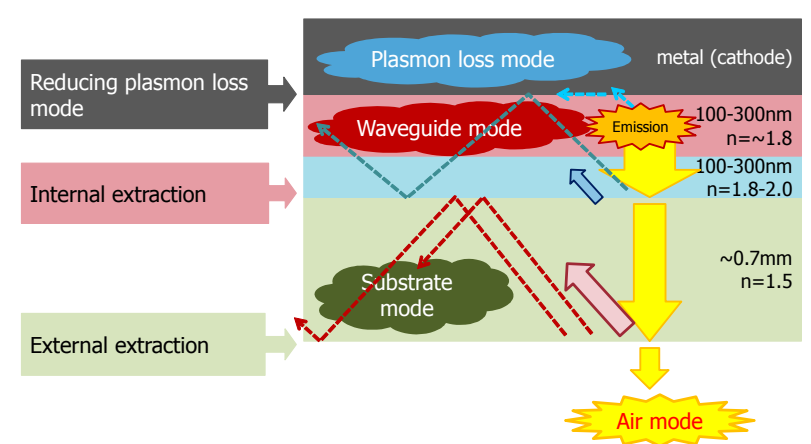

Fig. 4. Emission energy loss in OLED device

\subsection{Internal extraction structure}

It is known that the internal energy trapped in the stack and transparent electrode can be extracted by the scattering structure between substrate and electrode [13]. Significant advancements in efficacy can be made through internal light extraction techniques alone. However, integration of light extraction structures within the OLED structure presents additional challenges.

\begin{tabular}{|c|}
\hline Reflective electrode \\
\hline OLED stack $(\mathrm{n}=1.7-2.0)$ \\
\hline Transparent electrode $(\mathrm{n}=1.9-2.1)$ \\
\hline Planarization layer $(\mathrm{n}=1.7-2.0)$ \\
\hline Light scattering layer \\
\hline Substrate $(\mathrm{n}=1.5)$ \\
\hline External Extraction Structure (EES) \\
\hline
\end{tabular}

Fig. 5. OLED stack and optical indices with light extraction structures

We have developed a light extraction technology focused on extracting waveguide mode. A high refractive index scattering layer was imposed between substrate and transparent electrode for the extraction of waveguide mode (Fig. 5). This internal extraction structure (IES) is composed of two layers (Fig. 6), light scattering and planarization layers. The light scattering layer was fabricated on the substrate and was made from an optical transparent matrix material and scattering particles which possess different refractive indices from the matrix. The planarization layer was fabricated on the light scattering layer for planarizing the surface of the scattering layer to prevent the short circuit. This planarization layer was made of a high refractive index material.

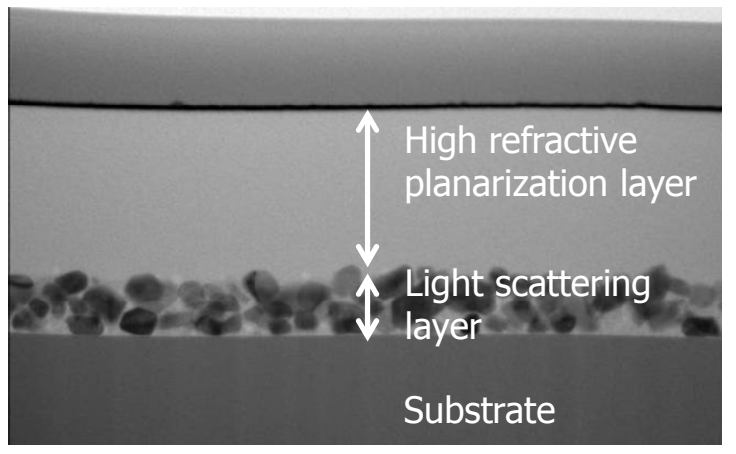

Fig. 6. Cross-section of TEM image 
The IES was optimally designed by go-it-alone optical simulations to maximize the extraction of light. Other characteristics that are necessary as the IES itself are scattering characteristics and low absorbance. These properties were optimized in the development of IES (Fig. 7). Also, as described above, the energy mode distribution largely depends on the stack design and materials. To increase the light extraction from the waveguide mode, several stack structures having larger waveguide mode were selected by optical simulations, and the optimum OLED stack structure with less influence on electrical characteristics was studied.

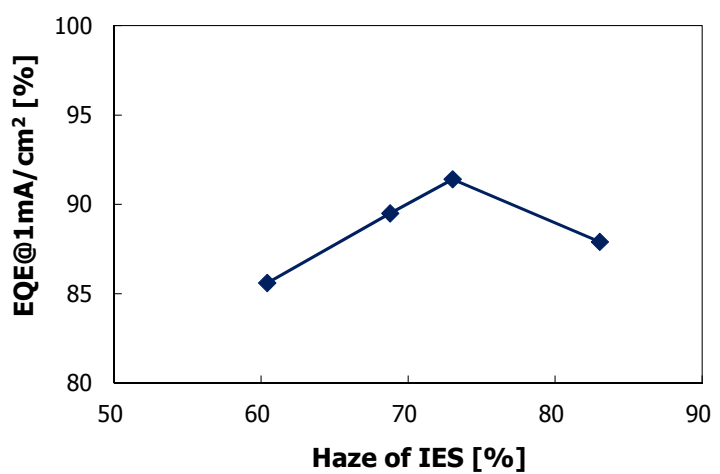

Fig. 7. Optimization of optical property on IES

\section{Designing of white OLEDs}

3.1.Performance of two-unit all-phosphorescent warm-white OLEDs

In order to optimize the light extraction and match the organic layer design, two-unit all-phosphorescent white OLEDs were investigated by original simulation. After experimental examinations of a light-emitting unit, the two-unit white OLED architecture with red-green (RG) and red-blue (RB) was selected. Moreover, the white OLED architecture with "Anode/RG/RB/Cathode" showed a higher EQE than that of "Anode/RB/RG/Cathode." (Fig. 8)

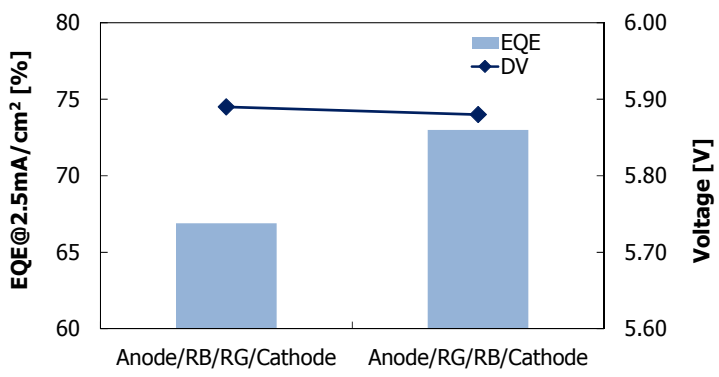

Fig. 8. Optimization of white OLED architecture
Optimal design of warm-white OLEDs along with IES has two light-emitting units, which are composed of RG and RB phosphorescent emission units. The light BE-2 (Figs. 1 and 3) was used as a blue phosphorescent emitter. In addition, we improved efficacy by decreasing driving voltage by using electron transport layer (ETL) that has high electron mobility.

A device with emission area of about 15 $\mathrm{cm}^{2}$, in which IES technology was introduced, was fabricated successfully. External extraction structure (EES) was applied onto the substrate to extract the substrate mode.

Performances of fabricated devices with emitting area of $15 \mathrm{~cm}^{2}$ are shown in Table 1 . Under operating condition of $1,000 \mathrm{~cd} / \mathrm{m}^{2}$, the device showed the power efficacy of 139 $1 \mathrm{~m} / \mathrm{W}$ with $2,857 \mathrm{~K}$ of CCT and CRI $=81$. The lifetime to LT50 at an initial luminance $\mathrm{L}_{0}=1,000 \mathrm{~cd} / \mathrm{m}^{2}$ is 65,000 hours. The luminance dependence on efficacy and the EQE are shown in Fig. 9.

Table 1. Performance of warm-white OLED

\begin{tabular}{lcc}
\hline \multicolumn{3}{c}{ Panel on Glass with IES } \\
\hline Efficacy & $\mathrm{Im} / \mathrm{W}$ & 139 \\
Luminance & $\mathrm{cd} / \mathrm{m}^{2}$ & 1,000 \\
EQE & $\%$ & 104.5 \\
Voltage & $\mathrm{V}$ & 5.3 \\
CCT & $\mathrm{K}$ & 2,857 \\
$1931 \mathrm{CIE}$ & $\mathrm{(x,y)}$ & $(0.47,0.44)$ \\
CRI & $\mathrm{Ra}$ & 81 \\
Estimated LT50 & $\mathrm{hrs}$ & $>65,000$ \\
Emissive area & $\mathrm{cm} 2$ & 15 \\
\hline
\end{tabular}

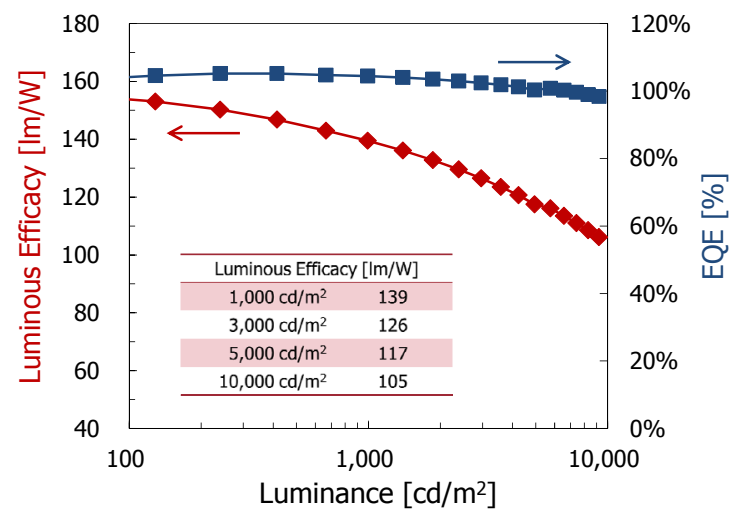

Fig. 9. Luminous efficacy and external quantum efficiency versus luminance 
Even at the high luminance level of 5,000 $\mathrm{cd} / \mathrm{m}^{2}$, the panel maintained efficacy over 110 $1 \mathrm{~m} / \mathrm{W}$, which is almost equivalent to LED package in the warm-white region. As for LED package, to make a lighting fixture, there are three losses in driver efficiency, thermal efficiency and optical efficiency [14]. Therefore, for a flat lighting fixture, LED efficacy drops to around half, and LED light bulb is estimated about $90 \mathrm{~lm} / \mathrm{W}$. On the other hand, as an OLED panel has only driver efficiency loss, the OLED fixture is estimated over $100 \mathrm{~lm} / \mathrm{W}$. We successfully demonstrated that the OLED has a competitive efficacy in warm-white region versus LEDs.

Angle dependence emission profile of the device is represented in Fig. 10. All three colors are close to the Lambertian emission pattern.

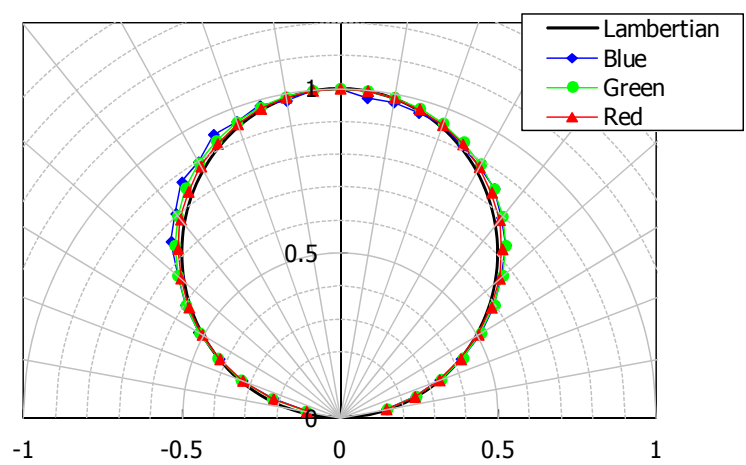

Fig. 10. Angle dependence emission profile

3.2 Performance of two-unit all-phosphorescent cool-white OLED

As described in section 2.1, we are developing deep-blue phosphorescent materials by applying our novel material design technology. However, the material design should be more difficult than that of the light-blue material due to having far higher triplet energy.

The device was fabricated in the same way as the warm-white white OLEDs except that the deep BE-1 (Fig. 3) was used as a blue phosphorescent emitter.

Under operating condition of $1,000 \mathrm{~cd} / \mathrm{m}^{2}$, the device showed power efficacy of 112 $1 \mathrm{~m} / \mathrm{W}$ with $3,722 \mathrm{~K}$. The luminance dependences on efficacy and EQE are shown in Fig. 11.

Fig. 12 shows electroluminescence (EL) spectra. Illumination quality is high, with CRI $=83$ and the special color rendering index R9 $=70$.

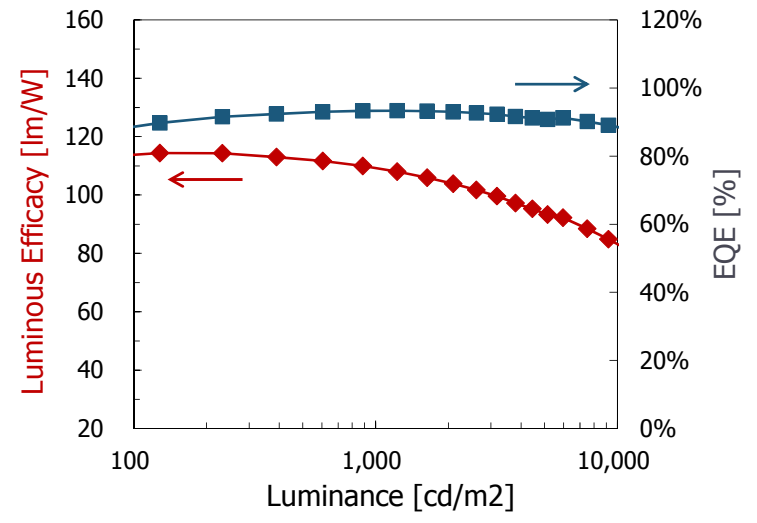

Fig. 11. Luminous efficacy and external quantum efficiency versus luminance

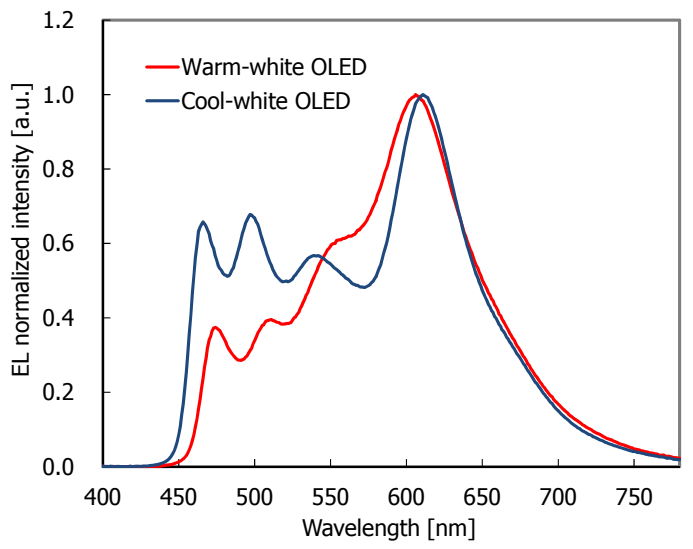

Fig. 12. Normalized EL spectra at $1,000 \mathrm{~cd} / \mathrm{m}^{2}$

\section{Conclusion}

The white OLED device with the world highest record of $139 \mathrm{~lm} / \mathrm{W}(\mathrm{CCT}=2857 \mathrm{~K})$ at $1,000 \mathrm{~cd} / \mathrm{m}^{2}$ was realized by introducing all-phosphorescent white OLED and light out-coupling technologies. The emission profile was very close to Lambertian and has negligible color change with viewing conditions. Even at the high luminance level of $5,000 \mathrm{~cd} / \mathrm{m}^{2}$, the panel maintained efficacy over $110 \mathrm{~lm} / \mathrm{W}$, which is almost equivalent to that of an LED package in the warm-white region. In addition, we have successfully demonstrated a white OLED panel with an extremely high efficacy of $112 \mathrm{~lm} / \mathrm{W}(\mathrm{CCT}=$ $3722 \mathrm{~K}$ ) at $1,000 \mathrm{~cd} / \mathrm{m}^{2}$ by using a new class of stable deep-blue phosphorescent emitters and related materials.

These outcomes would be a highly meaningful step to penetrate into the general lighting market.

\section{Acknowledgements}

The part of this work was supported by New Energy and Industrial Technology 
Development Organization (NEDO) through the "Fundamental Technology Development of Next Generation Lighting of High efficiency and High-quality" project. The authors also would like to thank Universal Display Corporation for Universal PHOLED $^{\mathrm{TM}}$ phosphorescent OLED technology.

\section{References}

1. C. W. Tang and S .A. VanSlyke, "Organic electroluminescent Diodes", Applied Physics Letters, 51 (1987) 913.

2. T. Tsujimura, S. Mizukoshi, N. Mori, K. Miwa, Y. Maekawa, M. Kohno, K. Onomura, K. Mameno, T. Anjiki, A. Kawakami and S. Van Slyke, "Scalable AMOLED Technologies for TV Application", IDW 08 Proceeding, (2008) 145.

3. C. Adachi, M. A. Baldo, M. E. Thompson and S. R. Forrest, "Nearly $100 \%$ internal phosphorescence efficiency in an organic light-emitting device", J. Appl. Phys., 90 (2001) 5048.

4. US Department of Energy, "Multi-year Program Plan, Solid-State Lighting Research and Development", (2013) 25.

5. D. G. Yoon, G. H. Bang, W. J. Hyun, H. J. Kong and B. D. Chin, "Solutionprocessed Light Extraction Structure and Metallic Grid Electrode for Enhanced Out coupling of OLED", SID 2014 Digest, (2014) 1571.

6. W. Youn, J. Lee, M. Xu, R. Singh and F. So, "Highly Efficient Phosphorescent OLEDs Fabricated on Corrugated High-Index-Refractive Substrates", SID 2014 Digest, (2014) 40.
7. H. Inoue, M. Fujita, K. Harai, H. Abe, T. Hori and M. Kashiwagi, "Improved Structure of Out-Coupling Film to Reduce the Angular Dependence of Chromaticity", SID 2012 Digest, (2012) 1507.

8. S. Mladenovski, K. Neyts, D. Pavicic, A. Werner and C. Rothe, "Exceptionally efficient organic light emitting devices using high refractive index substrates", Optics Express, 17 (2009) 7562.

9. H. H. Cho, B. Park, H. J. Kim, S. Jeon, J. H. Jeong and J. J. Kim, "Solutionprocessed photonic crystals to enhance the light outcoupling efficiency of organic light emitting diodes", Applied Optics, 49 (2010) 4024.

10. T. Tsujimura, K. Furukawa, H. Ii, H. Kashiwagi, M. Miyoshi, S. Mano, H. Araki and A. Ezaki, "World's First All Phosphorescence OLED Product for Lighting Application", IDW 2011 proceeding, (2011) 455 .

11. K. Furukawa, K. Kato and T. Iwasaki, "Recent progress of OLED performance for lighting application", IDW 13 Proceeding, (2013) 902.

12. H. Ito, K. Hiyama and H. Kita, "Development of Highly Efficient and Long-Lived Light-Blue Phosphorescent Material Technology", IDW 13 Proceeding, (2013) 860.

13. Y-S Tyan, Y. Q. Rao, J-S. Wang, R. Kesel, T. R. Cushman and W. J. Begley, "Fluorescent White OLED devices with Improved Light Extraction", SID 2008 Digest, (2008) 933.

14. US Department of Energy, "Multi-year Program Plan, Solid-State Lighting Research and Development", (2014) 46. 